\title{
Economic, Mental Health, HIV Prevention and HIV Treatment Impacts of COVID-19 and the COVID-19 Response on a Global Sample of Cisgender Gay Men and Other Men Who Have Sex with Men
}

\author{
Glenn-Milo Santos ${ }^{1,2}$ (D) Benjamin Ackerman ${ }^{3} \cdot$ Amrita Rao $^{4} \cdot$ Sara Wallach $^{4} \cdot$ George Ayala $^{5} \cdot$ Erik Lamontage $^{6,21}$. \\ Alex Garner ${ }^{7}$ Ian W. Holloway ${ }^{8}$. Sonya Arreola ${ }^{5}$. Vince Silenzio ${ }^{9}$. Susanne Strömdahl ${ }^{10,22}$. Louis Yu ${ }^{11}$. \\ Carol Strong ${ }^{12}$. Tyler Adamson ${ }^{4}$. Anna Yakusik ${ }^{6} \cdot \operatorname{Tran}_{\text {Thu Doan }}{ }^{13}$. Poyao Huang ${ }^{14}$. Damiano Cerasuolo ${ }^{15}$. \\ Amie Bishop ${ }^{16}$. Teymur Noori ${ }^{17}$. Anastasia Pharris ${ }^{17}$. Max Aung ${ }^{13}$. Masoud Dara ${ }^{18}$. Ssu Yu Chung ${ }^{19}$. \\ Marguerite Hanley ${ }^{20}$. Stefan Baral ${ }^{4}$. Chris Beyrer ${ }^{4}$. Sean Howell ${ }^{19}$
}

Published online: 11 July 2020

๑) Springer Science+Business Media, LLC, part of Springer Nature 2020

\begin{abstract}
There is an urgent need to measure the impacts of COVID-19 among gay men and other men who have sex with men (MSM). We conducted a cross-sectional survey with a global sample of gay men and other MSM ( $n=2732)$ from April 16, 2020 to May 4, 2020, through a social networking app. We characterized the economic, mental health, HIV prevention and HIV treatment impacts of COVID-19 and the COVID-19 response, and examined whether sub-groups of our study population are disproportionately impacted by COVID-19. Many gay men and other MSM not only reported economic and mental health consequences, but also interruptions to HIV prevention and testing, and HIV care and treatment services. These consequences were significantly greater among people living with HIV, racial/ethnic minorities, immigrants, sex workers, and socio-economically disadvantaged groups. These findings highlight the urgent need to mitigate the negative impacts of COVID-19 among gay men and other MSM.
\end{abstract}

Keywords COVID-19 $\cdot$ Economic impact $\cdot$ Mental health $\cdot$ HIV $\cdot$ AIDS $\cdot$ Gay $\cdot$ Men who have sex with men

\section{Resumen}

Existe una necesidad urgente para medir los impactos de COVID-19 entre hombres gay y otros hombres que tienen sexo con hombres (HSH). Hemos conducido una encuesta multifuncional con una prueba mundial de hombres gay y otros HSH $(\mathrm{n}=$ 2732) desde el 16 de Abril hasta el 4 de Mayo del 2020, a través de una aplicación de red social. Nosotros caracterizamos los impactos económicos, de salud mental, prevención del VIH y tratamiento del VIH e impactos a COVID-19 y la respuesta de COVID-19, y examinamos si subgrupos de nuestra población de estudio fueron impactados desproporcionadamente por COVID-19. Muchos hombres no tan solo reportaron consecuencias económicas y de salud mental, sino también interrupciones de prevención y de pruebas de VIH, y cuidado del VIH y servicios de tratamiento. Encontramos consecuencias más significantes entre personas viviendo con $\mathrm{VIH}$, grupos raciales/etnicos, migrantes, sexo servidores, y groupos socioeconomicamente disfavorecidos. Los resultados subrayan la necesidad crucial de mitigar los impactos multifacéticos de COVID-19 entre los hombres homosexuales y otros $\mathrm{HSH}$, especialmente para aquellos con vulnerabilidades entrelazadas.

Electronic supplementary material The online version of this article (https://doi.org/10.1007/s10461-020-02969-0) contains supplementary material, which is available to authorized users.

Glenn-Milo Santos

Glenn-Milo.Santos@ucsf.edu

Extended author information available on the last page of the article

\section{Introduction}

The COVID-19 pandemic poses a serious health threat worldwide, with over 4.4 million confirmed cases and over 300,000 deaths as of May 15, 2020 [1]. Globally efforts to curb the spread of COVID-19 have slowed the exponential growth of new cases, but have also led to unprecedented disruptions in society, with vast social, economic and health 
care consequences [2-5]. Unintended impacts of mitigation strategies to curb COVID-19 include exacerbating health disparities and deepening social inequities among marginalized groups including gay men and other men who have sex with men (MSM), MSM living with HIV, racial and ethnic minority MSM, and other vulnerable groups [6-9]. Indeed, emerging reports have documented the unique concerns and challenges experienced by gay men and other MSM during the COVID-19 pandemic, including mental health impacts resulting from anti-gay community backlash, arrests under false pretexts, and loss of privacy during contact tracing and monitoring for COVID-19 [10, 11]. Furthermore, many gay men and other MSM may be in industries that are more prone to COVID-19 disruptions, or at increased risk for unemployment, unstable housing, and food insecurity, making them more vulnerable to COVID-19's economic and health impacts compared to the general population [12-17]. Moreover, gay men and other MSM also have disproportionately higher unemployment rates relative to the general population [14, 18]. Therefore, COVID-19 disruptions can further heighten the economic barriers faced by many gay men and other MSM.

In addition, COVID-19 may amplify existing barriers that impede access to HIV prevention, testing, treatment, and care, potentially complicating slow-moving efforts to achieve global HIV targets among gay men and other MSM who remain disproportionately impacted by $\operatorname{HIV}[19,20]$. Gay men and other MSM are 22 times more likely to acquire HIV than the general population worldwide [21]. Reductions in access to effective HIV prevention tools including condoms, HIV testing, Pre-Exposure Prophylaxis (PrEP), and Post-Exposure Prophylaxis (PEP) for gay men and other MSM could increase HIV seroconversion, a life-altering event with no cure [21-23]. For gay men and other MSM living with HIV, unstructured treatment interruptions can lead to increased HIV viral load, lower CD4 count, HIV disease progression, and increased risk of developing an opportunistic infection [24, 25]. Such interruptions can also cause side effects, difficulties with adherence upon restarting HIV treatment, and even drug resistance [26-29]. Lastly, unsuppressed viral loads caused by treatment disruptions can contribute to increased risk of HIV transmission among partners of gay men and other MSM living with HIV [30, 31]. Hence, documenting how COVID-19 has impacted access to HIV prevention and testing, and HIV treatment and care services is of high public health importance.

There is an urgent need to examine the economic, mental health, HIV prevention and testing, and HIV treatment and care impacts of COVID-19 among gay men and other MSM in order to understand how this marginalized population is uniquely affected by this pandemic and the COVID-19 response, and to help inform the targeting of strategies to ameliorate these impacts. Furthermore, there is a need to examine whether sub-populations of gay men and other MSM are disproportionately impacted by COVID19, including among people living with HIV, racial/ethnic minorities, immigrants, sex workers, and socio-economically disadvantaged groups. We aim to fill the gaps on the economic and health impact of COVID-19 by conducting a study among a sample of gay men and other MSM living with or at high risk for HIV worldwide.

\section{Methods}

\section{Study Design}

This cross-sectional study is based on a COVID-19 Disparities survey implemented by the gay social networking app, Hornet. Hornet is a free, smart-phone based "Gay Social Networking" app with over 25 million users worldwide [32]. Therefore, we believe that the cisgender men who use this are gay men and other MSM. Hornet has been used as a means for conducting research on gay men and other MSM worldwide [20, 32-34]. Data collection for the current study occurred from April 16, 2020 to May 4, 2020, during which time Hornet users were invited to participate in a brief questionnaire with 58 questions regarding the impact of COVID-19 on economic vulnerability, mental health, HIV prevention, testing and treatment and care impacts. Hornet users were eligible to participate in the survey if they were age 18 and over and provided informed consent. A total of 4031 respondents participated; however, for this study, we have focused the analysis among participants who reported either being assigned male sex at birth or identify as intersex, and self-identify as male gender, and have available data on our outcomes and characteristics of interest $(n=2732)$. Study procedures were reviewed by the [Blinded for review] Institutional Review Board, which determined that the protocol qualified for Exempt status under Category 4.

\section{Measures}

Eligible, consenting participants responded to general demographic questions on age, country of residence, sex assigned at birth, gender identity, and sexual orientation. Participants were also asked about their HIV serostatus; membership to a racial or ethnic minority group (e.g., Do you consider yourself a member of an ethnic or racial minority?); immigrant status (e.g., "Are you, or is one or more of your parents, a migrant to the country in which you currently live?"); and history of engaging in sex work (e.g., "Have you ever engaged in sex work (i.e. being paid to have sex)"). 
Additionally, participants were asked a series of questions regarding the effects of COVID-19 on the following areas: (1) Economic Impacts; (2) Mental Health Impacts; (3) HIV Prevention and Testing Impacts; and (4) HIV Treatment and Care Impacts.

\section{Economic Impact Measures}

The survey assessed the impact of the COVID-19 pandemic on participants' economic status through questions regarding their employment status; ability to make ends meet (e.g., "Thinking of your household's total monthly income, is your household able to make ends meet?"); income level (e.g., "How much are you expecting your income to reduce because of the COVID-19 crisis?"); receipt of government financial assistance (e.g., "Are you receiving any additional financial benefits from work or government because of the COVID-19 crisis?"); loss of health insurance coverage (e.g., "Do you expect to lose your health insurance coverage because of the COVID-19 crisis?"); and food security (e.g., "Since the COVID-19 crisis began, have you had to cut the size of your meals or skip meals because there was not enough money for food?").

\section{Mental Health Impact Measures}

The survey also asked participants regarding their mental health using validated items from the Patient Health Questionnaire-4 (PHQ-4), a tool developed and validated by Kroenke, Spitzer, Williams and Löwe (2009) used to measure and screen for psychological distress, and particularly, depression and anxiety [35].

\section{HIV Prevention and Testing Impact Measures}

Participants at risk for HIV were asked whether they experienced changes in access to HIV prevention interventions including condoms, HIV in-person testing, HIV home testing, PrEP and PEP due to COVID-19, using Likert-type questions (e.g., "Do you feel you have access to HIV prevention strategies during the COVID-19 crisis?" with the following response options: "Definitely yes", "Probably yes", "Might or might not", "Probably not", "Definitely not"). These HIV prevention and testing questions were re-coded as binary to compare responses of "Definitely yes" to the other response options.

\section{HIV Treatment and Care Impact Measures}

For participants living with HIV, questions were also asked regarding their access to HIV providers (e.g., "Since the beginning of COVID-19 related social isolation in your country, by that or any other name, have you been able to see your HIV provider if you needed to?") and medication (e.g., "Do special measures related to COVID-19 impact your ability to access or refill your HIV medicine?"), as well as on their perception of being vulnerable to COVID-19 due to their HIV status (e.g., "Do you feel that you are more vulnerable to COVID-19 because you are living with HIV?").

\section{Data Analysis}

We performed descriptive analyses to characterize the economic, mental health, and HIV-prevention and HIV-care impacts of COVID-19 on survey participants. Outcomes were also stratified by key sociodemographic and clinical characteristics, including HIV status, being a racial/ethnic minority, immigrant status, health insurance coverage, and engaging in sex work. We examined between-group differences using Fisher's Exact and Chi-Squared tests, as appropriate, with a significance level of $\alpha=0.05$. For this study, analyses were conducted using a complete case approach, whereby only the fully-completed survey responses were included in the analyses. All analyses were conducted in R (R Foundation for Statistical Computing, Vienna, Austria).

\section{Results}

\section{Sample Characteristics}

We collected 2732 total responses from cisgender gay men and other MSM reported from 103 countries, with the largest numbers of responses from Brazil $(n=559)$, France $(\mathrm{n}=381)$, Mexico $(\mathrm{n}=181)$, Taiwan $(\mathrm{n}=177)$ and Russia $(\mathrm{n}=151)$ (see E-File Fig. 1). Most respondents identified as gay $(n=2294,84 \%)$, and about 1 in 5 respondents identified as a racial or ethnic minority $(n=485)$. Additionally, 274 respondents (11\%) reported having ever engaged in sex work, starting either before or after the pandemic. A total of 473 participants (17\%) reported that they are currently living with HIV. While the demographics of HIV-positive respondents were fairly similar to those who reported being HIV-negative, there was a greater proportion of individuals above the age of 50 living with HIV compared to those living without HIV ( $30 \%$ vs. $\left.19 \%, \chi^{2}=44.8, p<0.001\right)$. There was also a greater (though not statistically significant) proportion of individuals living with HIV who have reported engaging in sex work ( $14 \%$ vs. $10 \%, \chi^{2}=5.7, p=0.06$ ) (see E-File Table 1 for additional demographics information). 


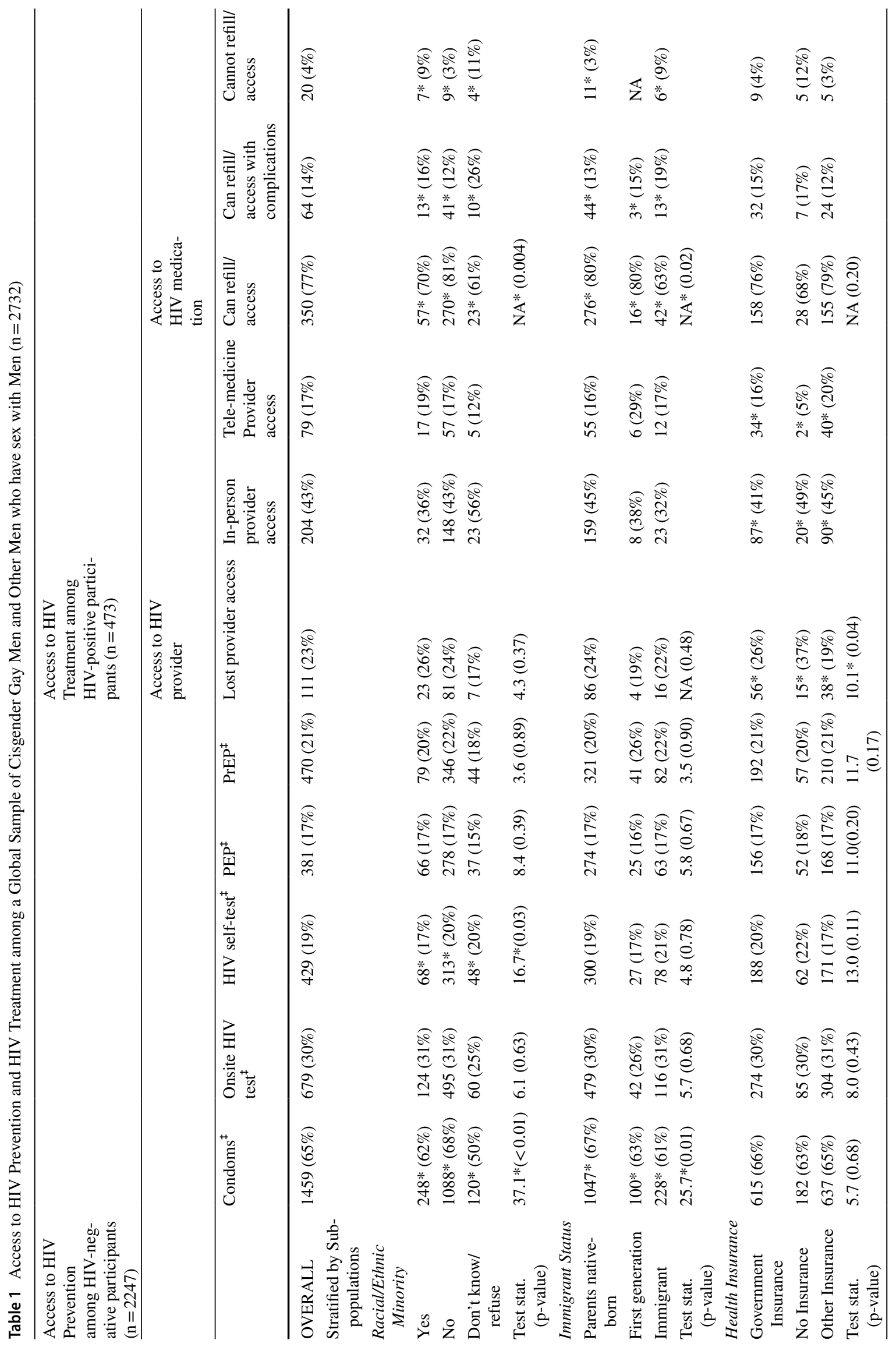




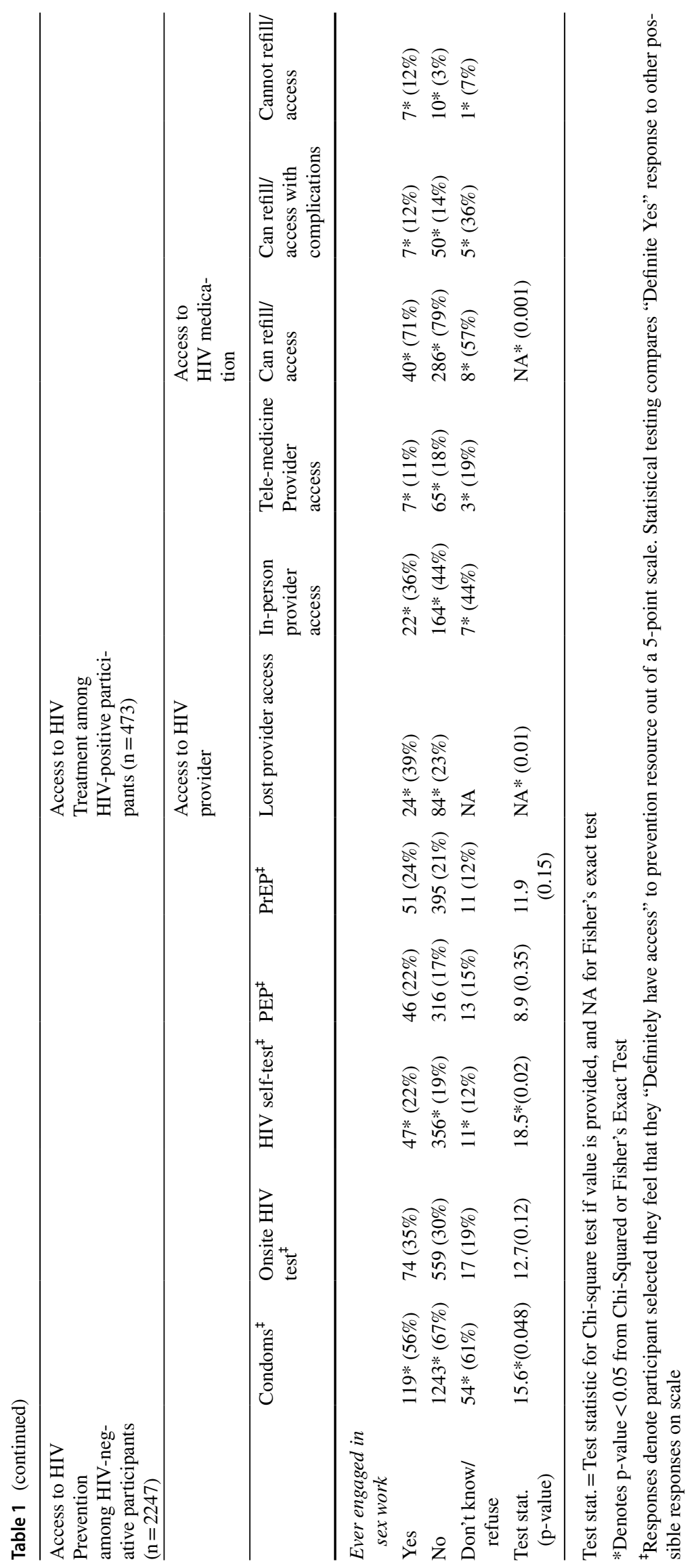




\section{Economic Impact}

Eleven percent of all participants reported losing employment as a result of changes due to COVID-19. Regardless of employment loss, a large proportion of participants (38\%) also indicated an inability to receive COVID-19-related financial benefits when needed, and $19 \%$ of participants reported reducing meal sizes or cutting meals completely due to COVID-19 (see E-File Table 2). Additionally, 4 out of every 10 respondents anticipated an income reduction of $30 \%$ or more due to COVID-19, and men living with HIV reported greater anticipated reductions in income compared to those not living with HIV ( $46 \%$ vs. $38 \% ; \chi^{2}=10.7$, $\mathrm{p}=0.01)$.

\section{Mental Health Impact}

COVID-19 has also had a substantial impact on the mental health of cisgender gay men and other MSM. Overall, $31 \%$ of participants reported moderate to severe psychological distress as measured by the PHQ-4 scale (16\% moderate, $15 \%$ severe). Based on the anxiety and depression subscales, 887 participants (35\%) screened positive for depression, and 856 participants (34\%) screened positive for anxiety. While these rates did not differ by HIV status, individuals who lost employment due to the pandemic screened positive for anxiety and depression at statistically significantly higher rates compared to those who did not: $50 \%$ of those who lost their jobs screened positive for depression compared to $31 \%$ of those who did not $(\chi 2=37.3, p<0.001)$, and $48 \%$ of those who lost their jobs screened positive for anxiety compared to $30 \%$ of those who did not $(\chi 2=35.9, \mathrm{p}<0.001)$. Additionally, $27.6 \%$ of participants who lost employment specifically reported feeling depressed nearly every day in the prior two weeks, compared to $11.4 \%$ of participants who did not lose employment $\left(\chi^{2}=65.5, \mathrm{p}<0.001\right)$. Similarly, $27.3 \%$ of those who became unemployed felt anxious nearly every day over the prior two weeks compared to $13.7 \%$ of participants who did not become unemployed $\left(\chi^{2}=42.2, \mathrm{p}<0.001\right)$ (see E-File Fig. 2).

\section{HIV Prevention Services and Testing Impacts}

More difficulties accessing prevention services were reported by study participants as a result of the COVID-19 pandemic (see Table 1). Among the 2247 participants not living with HIV, 1459 (65\%) felt they definitely still had access to condoms, though substantially fewer participants felt they had similar levels of access to onsite HIV testing (30\%), HIV athome testing (19\%), PrEP (21\%) or PEP (17\%). Respondents who identified as a racial or ethnic minority reported feeling less definite in their ability to access condoms (62\% vs. $68 \%$, $\left.\chi^{2}=37.1, p<0.001\right)$ and ability to access HIV self-tests ( $17 \%$ vs. $\left.20 \%, \chi^{2}=16.7, p=0.03\right)$ compared to those who did not identify as a racial or ethnic minority. Gay men and other MSM from immigrant backgrounds reported less definite access to condoms when compared to participants with parents who were born in their current country of residence ( $61 \%$ vs. $\left.67 \%, \chi^{2}=25.7, p=0.01\right)$. Less definite access to condoms was also reported by respondents who had ever engaged in sex work when compared to those who hadn't $\left(56 \%\right.$ vs. $\left.67 \%, \chi^{2}=15.6, p=0.048\right)$.

\section{HIV Care and Treatment Services Impacts}

Access to HIV treatment and care for study participants living with HIV has become difficult during the pandemic (see Table 1). Overall, 23\% of participants living with HIV indicated that they have lost access to their HIV providers as a result of COVID-19 social isolation measures, while $17 \%$ reported that they were able to communicate with providers via telemedicine. There were also significant differences in provider access by type of health insurance: uninsured individuals living with HIV reported greater rates of lost access to HIV providers compared to those with government insurance or other insurance types $\left(\chi^{2}=10.1, p=0.04\right)$. Out of the total number of respondents living with HIV, 455 (96\%) indicated that they were currently taking antiretroviral treatment (ART) for HIV. Of those taking ART, 18\% (around 1 in 5 ) indicated either an inability to refill or access their medication, or a difficulty in doing so. Additionally, individuals who identified as a racial or ethnic minority reported significantly more difficulties in accessing or refilling ART (25\%) compared to those who did not identify as a racial or ethnic minority (15\%) (Fisher's Exact $\mathrm{p}=0.004)$. Participants living with HIV who also reported having ever engaged in sex work reported significant challenges in accessing HIV treatment. Approximately 39\% reported losing access to their HIV provider (vs. 23\% among those who have not engaged in sex work) (Fisher's Exact $\mathrm{p}=0.01$ ), and $24 \%$ reported an inability or difficulty in refilling or accessing medication (vs. 17\% among those who have not engaged in sex work) (Fisher's Exact $\mathrm{p}=0.001$ ).

\section{Discussion}

COVID-19 and the efforts taken to stem its spread have resulted in interruptions to HIV prevention, testing and treatment services, severe economic consequences, and deleterious effects on mental health and quality of life among gay men and other MSM globally. These negative effects 
among gay men and other MSM are felt particularly strongly among sex workers, men belonging to racial/ethnic minority and immigrant groups, and men lacking financial means to access healthcare, reinforcing the intersectional vulnerabilities that pre-existed the COVID-19 pandemic.

Our findings highlight the severe economic impact experienced by gay men and other MSM worldwide due to COVID-19 and the response to this pandemic. Many gay men and other MSM have reported loss of employment and anticipated reductions in income. Gay men and other MSM living with HIV were also more likely to anticipate reductions in income compared to those not living with HIV. Furthermore, one in five gay men and other MSM have reported food insecurity as a result of COVID. Despite these negative economic consequences, about two in five gay men and other MSM indicated an inability to receive COVID-19-related financial benefits when needed. These economic impacts and unmet need for financial assistance will likely exacerbate existing disparities in employment and economic opportunity experienced by gay men and other MSM [11, 14, 18].

Among gay men and other MSM at high risk for HIV, a third report less than definite access to condoms, with those who identified as being part of a racial minority, reported being an immigrant, and those who engaged in sex work reporting significantly less access. The majority of gay men and other MSM not living with HIV also report less than definite access to HIV testing (seven out of ten for onsite HIV testing, and eight out of ten for HIV self-tests), with racial minorities reporting significantly less access to HIV self-tests. Moreover, more than four in five gay men and other MSM not living with HIV reported less than definite access to PrEP and PEP during the COVID-19 pandemic, with access for these HIV prevention tools being similar across all groups, including among racial minorities, immigrants, and people who engage in sex work. The low access for these HIV prevention tools due to COVID-19 observed are very alarming because gay men and other MSM continue to be disproportionately impacted by HIV worldwide, with prevalence of HIV for gay men and other MSM greatly exceeding the prevalence among the general population in many countries [21]. Furthermore, the disparities in access observed is also a great concern because it may exacerbate the heightened vulnerability to HIV among racial minority gay men and other MSM, immigrant gay men and other MSM and gay men and other MSM who engaged in sex work, driven by their exposure to structural risks and intersecting stigmas [36-40]. Additionally, it is incorrect to assume a diminished sexual risk for HIV among gay men and other MSM, especially for men in sero-different domestic partnerships or men who are employed as sex workers $[31,40]$. New data on from the U.S. has not only reinforced this point but also underscored the ongoing need for uninterrupted access to HIV prevention tools gay men and other
MSM may need [13]. Otherwise, consequences of the reductions in HIV prevention tools can be catastrophic in the HIV prevention response, particularly if they are not reversed.

In addition, among gay men and other MSM living with HIV, those who lack health insurance were more likely to experience disruptions in access to their HIV care during COVID-19. For example, one in five men living with HIV reported being unable to refill or access their HIV treatment medication during COVID-19, with those who identified as being part of a racial minority disproportionately affected. Lack of access to healthcare for other chronic health conditions (e.g. diabetes, hypertension) also presents a potential double jeopardy for participants with disrupted HIV care and prevention. As mentioned above, men living with HIV reported greater anticipated reductions in income as a result of the slowing of the economy and job loss due to the COVID-19 pandemic. The combination of the interruptions to care and reductions in income for gay men and other MSM living with HIV may also lead to subsequent barriers to HIV care, and in turn lead to treatment failure and increased HIV transmission [41-44]. Although currently, there is no evidence indicating that people living with HIV who are virologically suppressed are more vulnerable to acquiring COVID-19 or having greater severity of this illness than those not living with HIV, experts generally believe that those with high HIV viral load and low CD4 counts may be more susceptible to negative COVID-19 outcomes [45-47]. Therefore, to maintain the health of gay men and other MSM living with HIV and sustain the benefits from treatment as HIV prevention, efforts to reverse the HIV care and treatment disruptions occurring due to COVID-19 need to be implemented with the utmost urgency.

The results of this study highlight intersectional vulnerabilities related to HIV and COVID-19 among gay men and other MSM. Structural and social risk factors, like lack of health insurance and racial discrimination, have been shown to increase the risk of HIV acquisition and limit viral suppression [48-52]. Interruptions in care due to COVID-19 appear to be more acute in individuals with these same risk factors. Arguably, those who were already at greatest risk of poor HIV outcomes stand to be among the worst affected by this pandemic. In turn, poor immune functioning resulting from disruptions to HIV treatment, for example, may further vulnerabilities to COVID-19. There is a need to further understand these overlapping vulnerabilities among gay men and other MSM.

Our findings have several important implications. Clearly, interventions with the ability to circumvent the need to see patients in-person are needed to ensure delivery of HIV prevention and treatment services, maintain continuity of PrEP and ART treatment access (e.g., multi-month dispensing of treatment), mitigate new transmission events, and secure 
long-term health outcomes. Telemedicine may allow for sustained provider-patient interactions, even in the context of social distancing. Additionally, mobile health (mHealth) strategies, which have long been used to keep regular tabs on patients and ensure they are supported in order to maintain their health and well-being, will be even more important now that in-person interactions are limited [53]. In addition, strategies that limit the need for individuals to travel in order to access testing, medications, and other services will be needed, e.g., mobile delivery of medications, drivethrough testing, etc. Innovative strategies will be needed to (1) account for disparities in access to technology (both digital devices and internet access), and (2) monitor the efficacy and safety of medication use if regular testing and viral load monitoring are limited [53-55]. Importantly, our findings underscore the need to develop more robust strategies for sub-populations of gay men and other MSM-including racial and ethnic minorities, immigrants, the uninsured, those who engage in sex work-to narrow the disparities we observed. Specific targeted and tailored interventions designed with the unique needs of these sub-populations may be required to increase their access to economic support, as well as HIV prevention, care, treatment and mental health services. Individual-level targeted and tailored interventions are necessary, however, they are likely not sufficient. Ultimately, structural and policy changes that prioritize public health and address systemic barriers that maintain racism, xenophobia, and criminalization of sex work are required to alleviate the disparities observed in the long-term, and ensure economic and health equity among all gay men and other MSM.

Notably, there are some limitations of this study. First, individuals have to be users of Hornet to participate in the survey and therefore must have internet and smartphone access, which may limit the generalizability of the study findings to the target population of interest. This is a convenience sample and is not necessarily representative of all gay men and other MSM globally. Based on the sociodemographic characteristics of our sample, those engaged with the app and willing and able to take the time to fill out the questionnaire may likely be gay men and other MSM who are less affected by the negative consequences of COVID19. Therefore, it is possible that we may be underestimating the true magnitude of the challenges faced by gay men and other MSM as a result of COVID-19. Nevertheless, prior studies have also documented the ability of social networking platforms to efficiently reach hidden and stigmatized populations. Therefore, it is also plausible that this sample may have reached a more diverse group of gay men and other MSM compared to venue-based sampling or other convenience sampling strategies. Additionally, 892 individuals who initially agreed to take the survey left most of the survey incomplete and were therefore excluded. It is possible that certain factors, such as language barriers or stigma, may have led particular subgroups of these participants to not complete the survey, resulting in non-response bias. We are currently translating the survey into multiple languages to mitigate these limitations in future iterations of this study.

Another limitation stems from the limited information we collected regarding the factors that may be driving the disparities in access to services. Further studies, including qualitative interviews, are needed to explore the issues that may be contributing to the unequal levels of access and the cause of these disparities. Finally, the results rely on data that is cross-sectional in nature, which precludes our ability to examine temporal changes in the measures we analyzed.

Despite these limitations, our use of a rapid online survey among existing users of a social networking app provides a snapshot of the effects felt by gay men and other MSM in real-time, when it would otherwise be infeasible to collect new data. Key strengths of this dataset are, first, that it includes data on 2732 cisgender gay men and other MSM across 103 countries and, second, that it captures information on a range of domains that can be harnessed for future areas of research related to the implications of COVID-19, including individual financial security, health access, mental health, sex work, issues pertaining to immigration, domestic violence, and a range of others. Finally, while different countries are being impacted by COVID-19 at different times, these data also represent samples from some of the countries currently most affected by COVID-19, including Russia, Brazil, France and Mexico.

\section{Conclusion}

This study highlights COVID-19's negative impact on gay men and other MSM's access to HIV treatment and prevention services, as well as its economic consequences, and impact on mental health status among gay men and other MSM globally. The data presented underscore how COVID19 may function to deepen health disparities and social inequities by disproportionately impacting sub-groups of gay men and other MSM with intersecting vulnerabilities. As the COVID-19 pandemic continues, with vaccine development still in progress, it is imperative to expand efforts to mitigate the reductions in access to HIV prevention, testing, treatment and care services observed in this study, particularly among racial minority gay men and other MSM, immigrant gay men and other MSM, and gay men and other MSM who engage in sex work. It is also imperative to develop novel and creative strategies to support the health, economic security, and well-being of gay men and other MSM that can be deployed in the context of current efforts to curb the COVID-19 pandemic. Efforts are also needed to address the causes of economic and health disparities among sub-populations of gay 
men and other MSM with intersecting vulnerabilities, not just at the individual-level, but also at the structural level. In addition, continued monitoring of COVID-19's impact among gay men and other MSM is of great importance to better understand the evolving and longer-term impacts of the pandemic on this vulnerable population, and to ensure that responses are adjusted appropriately to sustain the gains made toward the goal of ending the HIV epidemic.

Acknowledgements We would like to acknowledge the following language translators: Ana Cara-Linda, Angelica Lopez, Anna Yakusik, Carol Strong, Damiano Cerasuolo, Edward Sutanto, Ezgi Ayeser, Henrique Vicentim, Howie Lim, Jose Garcia, Junming Wu, Ketty Rosenfeld, Luana Araujo, Mariano Ruiz, Maryam Motaghi, Omar Cherakoui, Panyaphon Phiphatkunarnon, Pedro Moreno, Poyao Huang, Souad Orhan, Stephane Ku, Tanat Chinbunchorn, Teak Sowaprux, Top Medping, Yalda Toofan. Authors also acknowledge support from members of the entire COVID Disparities Working Group: Benjamin Ackerman, Tyler Adamson, Ahmad Ahsan, Max Aung, George Ayala, Ron Balassanian, Stefan Baral, Sean Batir, Chris Beyrer, Amie Bishop, Damiano Cerasuolo, Tanat Chinbunchorn, Ssu Yu Chung, Clifton John Cortez, Phil Crehan, Masoud Dara, Vitaly Djuma, Tran Doan, Anna Mia Ekström, Alex Garner, Marguerite Hanley, Sean Howell, Poyao Huang, Adeeba Kamarulzaman, Maxim Kasianczuk, Dominik Koehler, Stephane Ku, Erik Lamontagne, Sin How Lim, Jonathan Lovitz, Greg Millett, Nur Afiqah Mohd Salleh, Vinh Nguyen, Tom Nichols, Teymur Noori, Michael Petrillo Alvarez, Nittaya Phanuphak Pungpapong, Anastasia Pharris Panyaphon Phiphatkunarnon, Ben Plumley, Amrita Rao, Glenn-Milo Santos, Vince Silenzio, Kyle Smith, Teak Sowaprux, Susanne Strömdahl, Carol Strong, Raina Tian, Annette Verster, Sara Wallach, Joshua Weaver, Louis Yu

Author Contributions GMS, BA, AR led the drafting of the manuscript and interpretation of results. BA conducted the data analyses. SH, SW, SB, CB, EL, IH, AG, GA, AB, TN, AP, MA, MH, LY, and TA developed the study collection instrument and procedures. All authors contributed to the interpretation of data, revised the manuscript for important intellectual content, and approved the final version of the manuscript.

\section{Compliance with Ethical Standards}

Conflict of interest All authors declare no conflicts of interest.

Ethical Approval Study procedures were reviewed by the Johns Hopkins Bloomberg School of Public Institutional Review Board, which determined that the protocol qualified for Exempt status under Category 4.

Informed Consent All participants provided consent for this study.

\section{References}

1. Dong E, Du H, Gardner L. An interactive web-based dashboard to track COVID-19 in real time. Lancet Infect Dis. 2020;20(5):533-4.

2. Yang H, Ma J. How an epidemic outbreak impacts happiness: factors that worsen (vs. protect) emotional well-being during the coronavirus pandemic. Psychiatry Res. 2020;289:113045.
3. Gonzalez-Sanguino C, Ausin B, AngelCastellanos M, Saiz J, Lopez-Gomez A, Ugidos C, et al. Mental Health Consequences during the Initial Stage of the 2020 Coronavirus Pandemic (COVID-19) in Spain. Brain Behav Immun. 2020.

4. Courtemanche C, Garuccio J, Le A, Pinkston J, Yelowitz A. Strong social distancing measures in the United States reduced the COVID-19 growth rate. Health Aff (Millwood). 2020:101377hlthaff202000608.

5. Kawohl W, Nordt C. COVID-19, unemployment, and suicide. Lancet Psychiatry. 2020;7(5):389-90.

6. Poteat T, Millett G, Nelson LE, Beyrer C. Understanding COVID19 Risks and Vulnerabilities among Black Communities in America: The Lethal Force of Syndemics. Annals of Epidemiology 2020.

7. Laurencin CT, McClinton A. The COVID-19 Pandemic: a Call to Action to Identify and Address Racial and Ethnic Disparities. J Racial Ethn Health Disparities. 2020.

8. Bibbins-Domingo K. This Time Must Be Different: Disparities During the COVID-19 Pandemic. Ann Intern Med. 2020.

9. Prevention CfDCa. Coronavirus Disease 2019 (COVID-19): U.S. Department of Health \& Human Services; 2020 [Available from: https://www.cdc.gov/coronavirus/2019-ncov/cases-updates/cases -in-us.html.

10. UNAIDS and MPact are extremely concerned about reports that LGBTI people are being blamed and abused during the COVID19 outbreak [press release]. 2020.

11. Bishop A. Vulnerability Amplified: The Impact of the COVID19 Pandemic on LGBTIQ People. OutRight Action International 2020.

12. Whittington C, Hadfield K, Calderón C. The Lives \& Livelihoods of Many in the LGBTQ Community Are At Risk Amidst COVID19 Crisis. Human Rights Campaign Foundation 2020.

13. Sanchez TH, Zlotorzynska M, Rai M, Baral SD. Characterizing the Impact of COVID-19 on Men Who Have Sex with Men Across the United States in April, 2020. AIDS Behav. 2020.

14. Charlton BM, Gordon AR, Reisner SL, Sarda V, Samnaliev M, Austin SB. Sexual orientation-related disparities in employment, health insurance, healthcare access and health-related quality of life: a cohort study of US male and female adolescents and young adults. BMJ Open. 2018;8(6):e020418.

15. Ecker J, Aubry T, Sylvestre J. A Review of the Literature on LGBTQ Adults Who Experience Homelessness. J Homosex. 2019;66(3):297-32323.

16. McCann E, Brown M. Homelessness among youth who identify as LGBTQ+: a systematic review. J Clin Nurs. 2019;28(11-12):2061-72.

17. Gonzalez-Pagan O. COVID-19 Resources for LGBTQ Employees (And Those Who Have Been Laid Off or Furloughed). New York, New York: Lambda Legal; 2020.

18. The Williams Institute. LGBT Demographic Data Interactive. UCLA School of Law: The Williams Institute; 2019.

19. Ayala G, Santos GM. Will the global HIV response fail gay and bisexual men and other men who have sex with men? J Int AIDS Soc. 2016;19(1):21098.

20. Ayala G, Santos GM, Arreola S, Garner A, Makofane K, Howell S. Blue-Ribbon Boys: factors associated with PrEP use, ART use and undetectable viral load among gay app users across six regions of the world. J Int AIDS Soc. 2018;21(Suppl 5):e25130.

21. UNAIDS. UNAIDS DATA. Geneva. Switzerland: UNAIDS; 2019. p. 2019.

22. Sullivan PS, Carballo-Dieguez A, Coates T, Goodreau SM, McGowan I, Sanders EJ, et al. Successes and challenges of HIV prevention in men who have sex with men. Lancet. 2012;380(9839):388-99. 
23. Beyrer C, Sullivan PS, Sanchez J, Dowdy D, Altman D, Trapence $\mathrm{G}$, et al. A call to action for comprehensive HIV services for men who have sex with men. Lancet. 2012;380(9839):424-38.

24. Strategies for Management of Antiretroviral Therapy Study G, El-Sadr WM, Lundgren J, Neaton JD, Gordin F, Abrams D, et al. CD4+ count-guided interruption of antiretroviral treatment. $\mathrm{N}$ Engl J Med. 2006;355(22):2283-96.

25. Samji H, Taha TE, Moore D, Burchell AN, Cescon A, Cooper C, et al. Predictors of unstructured antiretroviral treatment interruption and resumption among HIV-positive individuals in Canada. HIV Med. 2015;16(2):76-877.

26. TenoRes Study G. Global epidemiology of drug resistance after failure of WHO recommended first-line regimens for adult HIV-1 infection: a multicentre retrospective cohort study. Lancet Infect Dis. 2016;16(5):565-75.

27. Schweighardt B, Ortiz GM, Grant RM, Wellons M, Miralles GD, Kostrikis LG, et al. Emergence of drug-resistant HIV-1 variants in patients undergoing structured treatment interruptions. AIDS. 2002;16(17):2342-4.

28. Bansi-Matharu L, Rodriguez Loria G, Cole SR, Mugerwa H, Vecino I, Lundgren J, et al. Risk factors for antiretroviral therapy (ART) discontinuation in a large multinational trial of early ART initiators. AIDS. 2019;33(8):1385-90.

29. Mann M, Lurie MN, Kimaiyo S, Kantor R. Effects of political conflict-induced treatment interruptions on HIV drug resistance. AIDS Rev. 2013;15(1):15-24.

30. Cohen MS, Chen YQ, McCauley M, Gamble T, Hosseinipour MC, Kumarasamy N, et al. Antiretroviral therapy for the prevention of HIV-1 transmission. N Engl J Med. 2016;375(9):830-9.

31. Rodger AJ, Cambiano V, Bruun T, Vernazza P, Collins S, Degen $\mathrm{O}$, et al. Risk of HIV transmission through condomless sex in serodifferent gay couples with the HIV-positive partner taking suppressive antiretroviral therapy (PARTNER): final results of a multicentre, prospective, observational study. Lancet. 2019;393(10189):2428-38

32. Bourne A, Alba B, Garner A, Spiteri G, Pharris A, Noori T. Use of, and likelihood of using, HIV pre-exposure prophylaxis among men who have sex with men in Europe and Central Asia: findings from a 2017 large geosocial networking application survey. Sex Transm Infect. 2019;95(3):187-92.

33. Baral S, Turner RM, Lyons CE, Howell S, Honermann B, Garner A, et al. Population size estimation of gay and bisexual men and other men who have sex with men using social media-based platforms. JMIR Public Health Surveill. 2018;4(1):e15.

34. Torres TS, Luz PM, De Boni RB, de Vasconcellos MTL, Hoagland B, Garner A, et al. Factors associated with PrEP awareness according to age and willingness to use HIV prevention technologies: the 2017 online survey among MSM in Brazil(.). AIDS Care. 2019;31(10):1193-202.

35. Kroenke K, Spitzer RL, Williams JB, Lowe B. An ultra-brief screening scale for anxiety and depression: the PHQ-4. Psychosomatics. 2009;50(6):613-21.

36. Santos GM, Do T, Beck J, Makofane K, Arreola S, Pyun T, et al. Syndemic conditions associated with increased HIV risk in a global sample of men who have sex with men. Sex Transm Infect. 2014;90(3):250-3.

37. Martinez O, Brady KA, Levine E, Page KR, Zea MC, Yamanis TJ, et al. Using syndemics theory to examine HIV sexual risk among latinx men who have sex with men in Philadelphia, PA: findings from the National HIV Behavioral Surveillance. EHQUIDAD. 2020;13:217-36

38. Santos GM, Makofane K, Arreola S, Do T, Ayala G. Reductions in access to HIV prevention and care services are associated with arrest and convictions in a global survey of men who have sex with men. Sex Transm Infect. 2017;93(1):62-4.
39. Arreola S, Santos GM, Beck J, Sundararaj M, Wilson PA, Hebert $\mathrm{P}$, et al. Sexual stigma, criminalization, investment, and access to HIV services among men who have sex with men worldwide. AIDS Behav. 2015;19(2):227-34.

40. Baral SD, Friedman MR, Geibel S, Rebe K, Bozhinov B, Diouf D, et al. Male sex workers: practices, contexts, and vulnerabilities for HIV acquisition and transmission. Lancet. 2015;385(9964):260-73.

41. Delpierre C, Cuzin L, Lauwers-Cances V, Datta GD, Berkman L, Lang T. Unemployment as a risk factor for AIDS and death for HIV-infected patients in the era of highly active antiretroviral therapy. Sex Transm Infect. 2008;84(3):183-6.

42. Maulsby C, Parker LJ, White JJ, Latkin CA, Mugavero MJ, Flynn $\mathrm{CP}$, et al. HIV and employment among Black men who have sex with men in Baltimore. AIDS Care. 2020;32(6):735-43.

43. Maulsby CH, Ratnayake A, Hesson D, Mugavero MJ, Latkin CA. A Scoping Review of Employment and HIV. AIDS Behav. 2020.

44. Conyers LM, Richardson LA, Datti PA, Koch LC, Misrok M. A Critical review of health, social, and prevention outcomes associated with employment for people living With HIV. AIDS Educ Prev. 2017;29(5):475-90.

45. Richardson S, Hirsch JS, Narasimhan M, Crawford JM, McGinn T, Davidson KW, et al. Presenting Characteristics, Comorbidities, and Outcomes Among 5700 Patients Hospitalized With COVID19 in the New York City Area. JAMA. 2020.

46. Laurence J. Why Aren't People Living with HIV at Higher Risk for Developing Severe Coronavirus Disease 2019 (COVID-19)? AIDS Patient Care STDS. 2020

47. Hodson M. Coronavirus (COVID-19) and HIV. AIDSMAP. 2020.

48. Earnshaw VA, Bogart LM, Dovidio JF, Williams DR. Stigma and racial/ethnic HIV disparities: moving toward resilience. Am Psychol. 2013;68(4):225-36.

49. Han CS, Ayala G, Paul JP, Boylan R, Gregorich SE, Choi KH. Stress and coping with racism and their role in sexual risk for HIV among African American, Asian/Pacific Islander, and Latino men who have sex with men. Arch Sex Behav. 2015;44(2):411-20.

50. Mizuno Y, Borkowf CB, Ayala G, Carballo-Dieguez A, Millett GA. Correlates of sexual risk for HIV among US-born and foreign-born Latino men who have sex with men (MSM): an analysis from the Brothers y Hermanos study. J Immigr Minor Health. 2015;17(1):47-55.

51. Wood S, Ratcliffe S, Gowda C, Lee S, Dowshen NL, Gross R. Impact of insurance coverage on HIV transmission potential among antiretroviral therapy-treated youth living with HIV. AIDS. 2018;32(7):895-902.

52. Riley ED, Moore KL, Haber S, Neilands TB, Cohen J, Kral AH. Population-level effects of uninterrupted health insurance on services use among HIV-positive unstably housed adults. AIDS Care. 2011;23(7):822-30.

53. Gras G. Use of telemedicine in the management of infectious diseases. Med Mal Infect. 2018;48(4):231-7.

54. Catalani C, Philbrick W, Fraser H, Mechael P, Israelski DM. mHealth for HIV treatment \& prevention: a systematic review of the literature. Open AIDS J. 2013;7:17-411.

55. Rana AI, van den Berg JJ, Lamy E, Beckwith CG. Using a mobile health intervention to support HIV treatment adherence and retention among patients at risk for disengaging with care. AIDS Patient Care STDS. 2016;30(4):178-84.

Publisher's Note Springer Nature remains neutral with regard to jurisdictional claims in published maps and institutional affiliations. 


\section{Affiliations}

\section{Glenn-Milo Santos ${ }^{1,2}$ (D) Benjamin Ackerman ${ }^{3} \cdot$ Amrita Rao $^{4} \cdot$ Sara Wallach $^{4} \cdot$ George Ayala $^{5}$. Erik Lamontage ${ }^{6,21}$. Alex Garner ${ }^{7} \cdot \operatorname{lan}$ W. Holloway ${ }^{8}$. Sonya Arreola ${ }^{5}$. Vince Silenzio ${ }^{9}$. Susanne Strömdahl ${ }^{10,22}$. Louis Yu $^{11}$. Carol Strong ${ }^{12}$. Tyler Adamson ${ }^{4}$. Anna Yakusik ${ }^{6} \cdot$ Tran Thu Doan $^{13}$. Poyao Huang ${ }^{14}$. Damiano Cerasuolo ${ }^{15}$. Amie Bishop ${ }^{16}$. Teymur Noori ${ }^{17}$. Anastasia Pharris ${ }^{17}$. Max Aung ${ }^{13}$. Masoud Dara ${ }^{18}$. Ssu Yu Chung ${ }^{19}$. Marguerite Hanley ${ }^{20}$. Stefan Baral ${ }^{4}$. Chris Beyrer ${ }^{4}$ Sean Howell ${ }^{19}$}

1 Community Health Systems Department, University of California San Francisco, San Francisco, CA, USA

2 Center of Public Health Research, San Francisco Department of Public Health, San Francisco, CA, USA

3 Department of Biostatistics, Johns Hopkins Bloomberg School of Public Health, Baltimore, MD, USA

4 Center for Public Health and Human Rights, Johns Hopkins Bloomberg School of Public Health, Baltimore, MD, USA

5 MPact Global Action for Gay Men's Health and Rights, Oakland, CA, USA

6 Joint United Nations Programme On HIV and AIDS, Geneva, Switzerland

7 Hornet, Los Angeles, CA, USA

8 Luskin School of Public Affairs, University of California Los Angeles, Los Angeles, CA, USA

9 Rutgers School of Public Health, Piscataway, NJ, USA

10 Department of Global Public Health, Karolinska Institutet, Stockholm, Sweden

11 Department of Mathematics, Computer Science, and Statistics, Gustavus Adolphus College, Saint Peter, MN, USA
12 Department of Public Health, National Cheng Kung University, Tainan City, Taiwan

13 Department of Biostatistics, School of Public Health, University of Michigan, Ann Arbor, MI, USA

14 Department of Communication, University of California San Diego, San Diego, CA, USA

15 University Hospital of Caen, Caen, France

16 OutRight Action International, New York, NY, USA

17 European Centre for Disease Prevention and Control, Solna, Sweden

18 WHO Regional Office for Europe, Copenhagen, Denmark

19 LGBT Foundation, San Francisco, CA, USA

20 Tech4HIV, San Francisco, CA, USA

21 Aix-Marseille University, CNRS, EHESS, Centrale Marseille, AMSE, Chemin du Château Lafarge, 13290 Les Milles, France

22 Section of Infectious Diseases, Department of Medical Sciences, Uppsala University, Uppsala, Sweden 